\title{
Optimal bounds for Neuman means in terms of geometric, arithmetic and quadratic means
}

Wei-Mao Qian ${ }^{1}$ and Yu-Ming Chu ${ }^{2^{*}}$

${ }^{*}$ Correspondence:

chuyuming2005@126.com

${ }^{2}$ School of Mathematics and Computation Science, Hunan City

University, Yiyang, 413000, China Full list of author information is

available at the end of the article

\section{Abstract}

In this paper, we present sharp bounds for the two Neuman means $S_{H A}$ and $S_{C A}$ derived from the Schwab-Borchardt mean in terms of convex combinations of either the weighted arithmetic and geometric means or the weighted arithmetic and quadratic means, and the mean generated either by the geometric or by the quadratic mean.

MSC: $26 \mathrm{E} 60$

Keywords: Schwab-Borchardt mean; Neuman mean; geometric mean; arithmetic mean; quadratic mean

\section{Introduction}

Let $a, b>0$ with $a \neq b$, then the Schwab-Borchardt mean $S B(a, b)$ is defined by

$$
S B(a, b)= \begin{cases}\frac{\sqrt{b^{2}-a^{2}}}{\cos ^{-1}(a / b)}, & a<b, \\ \frac{\sqrt{a^{2}-b^{2}}}{\cosh ^{-1}(a / b)}, & a>b,\end{cases}
$$

where $\cos ^{-1}(x)$ and $\cosh ^{-1}(x)=\log \left(x+\sqrt{x^{2}-1}\right)$ are the inverse cosine and inverse hyperbolic cosine functions, respectively.

It is well known that $S B(a, b)$ is strictly increasing in both $a$ and $b$, nonsymmetric and homogeneous of degree 1 with respect to $a$ and $b$. Many symmetric bivariate means are special cases of the Schwab-Borchardt mean, for example,

$$
\begin{aligned}
& P(a, b)=\frac{a-b}{2 \sin ^{-1}[(a-b) /(a+b)]}=S B(G, A) \quad \text { is the first Seiffert mean, } \\
& T(a, b)=\frac{a-b}{2 \tan ^{-1}[(a-b) /(a+b)]}=S B(A, Q) \quad \text { is the second Seiffert mean, } \\
& M(a, b)=\frac{a-b}{2 \sinh ^{-1}[(a-b) /(a+b)]}=S B(Q, A) \quad \text { is the Neuman-Sándor mean, } \\
& L(a, b)=\frac{a-b}{2 \tanh ^{-1}[(a-b) /(a+b)]}=S B(A, G) \quad \text { is the logarithmic mean, }
\end{aligned}
$$

where $G(a, b)=\sqrt{a b}, A(a, b)=(a+b) / 2$ and $Q(a, b)=\sqrt{\left(a^{2}+b^{2}\right) / 2}$ denote the classical geometric mean, arithmetic mean and quadratic mean of $a$ and $b$, respectively. The SchwabBorchardt mean $S B(a, b)$ was investigated in $[1,2]$.

@2014 Qian and Chu; licensee Springer. This is an Open Access article distributed under the terms of the Creative Commons Attribution License (http://creativecommons.org/licenses/by/2.0), which permits unrestricted use, distribution, and reproduction in any medium, provided the original work is properly cited. 
Let $H(a, b)=2 a b /(a+b), C(a, b)=\left(a^{2}+b^{2}\right) /(a+b)$ be the harmonic and contraharmonic means of two positive numbers $a$ and $b$, respectively. Then it is well known that

$$
\begin{aligned}
H(a, b) & <G(a, b)<L(a, b)<P(a, b) \\
& <A(a, b)<M(a, b)<T(a, b)<Q(a, b)<C(a, b)
\end{aligned}
$$

for $a, b>0$ with $a \neq b$.

Recently, the Schwab-Borchardt mean and its special cases have been the subject of intensive research. Neuman and Sándor [3, 4] proved that the inequalities

$$
\begin{aligned}
& P(a, b)>\frac{2}{\pi} A(a, b), \quad \frac{A(a, b)}{\log (1+\sqrt{2})}>M(a, b)>\frac{\pi}{4 \log (1+\sqrt{2})} T(a, b), \\
& T(A(a, b), G(a, b))<P(a, b), \quad T(a, b)>T(A(a, b), Q(a, b)), \\
& L(a, b)<L(A(a, b), G(a, b)), \quad M(a, b)<L(A(a, b), Q(a, b)), \\
& L(a, b)>H(P(a, b), G(a, b)), \quad P(a, b)>H(L(a, b), A(a, b)), \\
& M(a, b)>H(T(a, b), A(a, b)), \quad T(a, b)>H(M(a, b), Q(a, b)), \\
& G(a, b) P(a, b)<L^{2}(a, b)<\frac{G^{2}(a, b)+P^{2}(a, b)}{2} \\
& L(a, b) A(a, b)<P^{2}(a, b)<\frac{L^{2}(a, b)+A^{2}(a, b)}{2} \\
& A(a, b) T(a, b)<M^{2}(a, b)<\frac{A^{2}(a, b)+T^{2}(a, b)}{2}, \\
& M(a, b) Q(a, b)<T^{2}(a, b)<\frac{M^{2}(a, b)+Q^{2}(a, b)}{2} \\
& Q^{1 / 3}(a, b) A^{2 / 3}(a, b)<M(a, b)<\frac{1}{3} Q(a, b)+\frac{2}{3} A(a, b)
\end{aligned}
$$

hold for all $a, b>0$ with $a \neq b$. In [5], the author proved that the double inequalities

$$
\alpha Q(a, b)+(1-\alpha) A(a, b)<M(a, b)<\beta Q(a, b)+(1-\beta) A(a, b)
$$

and

$$
\lambda C(a, b)+(1-\lambda) A(a, b)<M(a, b)<\mu C(a, b)+(1-\mu) A(a, b)
$$

hold for all $a, b>0$ with $a \neq b$ if and only if $\alpha \leq[1-\log (1+\sqrt{2})] /[(\sqrt{2}-1) \log (1+\sqrt{2})]=$ $0.3249 \ldots, \beta \geq 1 / 3, \lambda \leq[1-\log (1+\sqrt{2})] / \log (1+\sqrt{2})=0.1345 \ldots$ and $\mu \geq 1 / 6$. Chu and Long [6] found that the double inequality

$$
M_{p}(a, b)<M(a, b)<q I(a, b)
$$

holds for all $a, b>0$ with $a \neq b$ if and only if $p \leq \log 2 / \log [2 \log (1+\sqrt{2})]=1.224 \ldots$ and $q \geq$ $e /[2 \log (1+\sqrt{2})]=1.5420 \ldots$, where $M_{p}(a, b)=\left[\left(a^{p}+b^{p}\right) / 2\right]^{1 / p}(p \neq 0)$ and $M_{0}(a, b)=\sqrt{a b}$ is the $p$ th power mean of $a$ and $b$. Zhao et al. [7] presented the least values $\alpha_{1}, \alpha_{2}, \alpha_{3}$ and 
the greatest values $\beta_{1}, \beta_{2}, \beta_{3}$ such that the double inequalities

$$
\begin{aligned}
& \alpha_{1} H(a, b)+\left(1-\alpha_{1}\right) Q(a, b)<M(a, b)<\beta_{1} H(a, b)+\left(1-\beta_{1}\right) Q(a, b), \\
& \alpha_{2} G(a, b)+\left(1-\alpha_{2}\right) Q(a, b)<M(a, b)<\beta_{2} G(a, b)+\left(1-\beta_{2}\right) Q(a, b)
\end{aligned}
$$

and

$$
\alpha_{3} H(a, b)+\left(1-\alpha_{3}\right) C(a, b)<M(a, b)<\beta_{3} H(a, b)+\left(1-\beta_{3}\right) C(a, b)
$$

hold for all $a, b>0$ with $a \neq b$.

Very recently, the bivariate means $S_{A H}, S_{H A}, S_{C A}$ and $S_{A C}$ derived from the SchwabBorchardt mean have been defined by Neuman $[8,9]$ as follows:

$$
S_{A H}=S B(A, H), \quad S_{H A}=S B(H, A), \quad S_{C A}=S B(C, A), \quad S_{A C}=S B(A, C) .
$$

We call the means $S_{A H}, S_{H A}, S_{C A}$ and $S_{A C}$ given in (1.3) the Neuman means. Moreover, let $v=(a-b) /(a+b) \in(-1,1)$, then the following explicit formulas for $S_{A H}, S_{H A}, S_{A C}$ and $S_{C A}$ have been found by Neuman [8]:

$$
\begin{array}{ll}
S_{A H}=A \frac{\tanh (p)}{p}, & S_{H A}=A \frac{\sin (q)}{q}, \\
S_{C A}=A \frac{\sinh (r)}{r}, & S_{A C}=A \frac{\tan (s)}{s},
\end{array}
$$

where $p, q, r$ and $s$ are defined implicitly as $\operatorname{sech}(p)=1-v^{2}, \cos (q)=1-v^{2}, \cosh (r)=1+v^{2}$ and $\sec (s)=1+v^{2}$, respectively. Clearly, $p \in(0, \infty), q \in(0, \pi / 2), r \in(0, \log (2+\sqrt{3}))$ and $s \in(0, \pi / 3)$.

In [8], Neuman proved that the inequalities

$$
\begin{aligned}
& H(a, b)<S_{A H}(a, b)<L(a, b)<S_{H A}(a, b)<P(a, b), \\
& T(a, b)<S_{C A}(a, b)<Q(a, b)<S_{A C}(a, b)<C(a, b)
\end{aligned}
$$

hold for $a, b>0$ with $a \neq b$.

He et al. [10] found the greatest values $\alpha_{1}, \alpha_{2} \in[0,1 / 2], \alpha_{3}, \alpha_{4} \in[1 / 2,1]$ and the least values $\beta_{1}, \beta_{2} \in[0,1 / 2], \beta_{3}, \beta_{4} \in[1 / 2,1]$ such that the double inequalities

$$
\begin{aligned}
& H\left(\alpha_{1} a+\left(1-\alpha_{1}\right) b, \alpha_{1} b+\left(1-\alpha_{1}\right) a\right)<S_{A H}(a, b)<H\left(\beta_{1} a+\left(1-\beta_{1}\right) b, \beta_{1} b+\left(1-\beta_{1}\right) a\right) \\
& H\left(\alpha_{2} a+\left(1-\alpha_{2}\right) b, \alpha_{2} b+\left(1-\alpha_{2}\right) a\right)<S_{H A}(a, b)<H\left(\beta_{2} a+\left(1-\beta_{2}\right) b, \beta_{2} b+\left(1-\beta_{2}\right) a\right) \\
& C\left(\alpha_{3} a+\left(1-\alpha_{3}\right) b, \alpha_{3} b+\left(1-\alpha_{3}\right) a\right)<S_{C A}(a, b)<C\left(\beta_{3} a+\left(1-\beta_{3}\right) b, \beta_{3} b+\left(1-\beta_{3}\right) a\right)
\end{aligned}
$$

and

$$
C\left(\alpha_{4} a+\left(1-\alpha_{4}\right) b, \alpha_{4} b+\left(1-\alpha_{4}\right) a\right)<S_{A C}(a, b)<C\left(\beta_{4} a+\left(1-\beta_{4}\right) b, \beta_{4} b+\left(1-\beta_{4}\right) a\right)
$$

hold for all $a, b>0$ with $a \neq b$. 
It follows from (1.2) and (1.6) together with (1.7) that

$$
G(a, b)<S_{H A}(a, b)<A(a, b)<S_{C A}(a, b)<Q(a, b)
$$

for all $a, b>0$ with $a \neq b$.

For fixed $a, b>0$ with $a \neq b$, let $x \in[0,1 / 2], y \in[1 / 2,1]$,

$$
\begin{aligned}
& f(x)=G[x a+(1-x) b, x b+(1-x) a], \\
& g(y)=Q[y a+(1-y) b, y b+(1-y) a] .
\end{aligned}
$$

Then it is not difficult to verify that $f(x)$ and $g(y)$ are continuous and strictly increasing on $[0,1 / 2]$ and $[1 / 2,1]$, respectively. Note that

$$
\begin{aligned}
& f(0)=G(a, b)<S_{H A}(a, b)<A(a, b)=f(1 / 2), \\
& g(1 / 2)=A(a, b)<S_{C A}(a, b)<Q(a, b)=g(1) .
\end{aligned}
$$

Motivated by (1.8)-(1.12), in the article we present the best possible parameters $\alpha_{1}, \alpha_{2}, \beta_{1}$, $\beta_{2} \in \mathbb{R}, \alpha_{3}, \beta_{3} \in[0,1 / 2]$ and $\alpha_{4}, \beta_{4} \in[1 / 2,1]$ such that the double inequalities

$$
\begin{aligned}
& \alpha_{1} A(a, b)+\left(1-\alpha_{1}\right) G(a, b)<S_{H A}(a, b)<\beta_{1} A(a, b)+\left(1-\beta_{1}\right) G(a, b), \\
& \alpha_{2} A(a, b)+\left(1-\alpha_{2}\right) Q(a, b)<S_{C A}(a, b)<\beta_{2} A(a, b)+\left(1-\beta_{2}\right) Q(a, b), \\
& G\left[\alpha_{3} a+\left(1-\alpha_{3}\right) b, \alpha_{3} b+\left(1-\alpha_{3}\right) a\right]<S_{H A}(a, b)<G\left[\beta_{3} a+\left(1-\beta_{3}\right) b, \beta_{3} b+\left(1-\beta_{3}\right) a\right], \\
& Q\left[\alpha_{4} a+\left(1-\alpha_{4}\right) b, \alpha_{4} b+\left(1-\alpha_{4}\right) a\right]<S_{C A}(a, b)<Q\left[\beta_{4} a+\left(1-\beta_{4}\right) b, \beta_{4} b+\left(1-\beta_{4}\right) a\right]
\end{aligned}
$$

hold for all $a, b>0$ with $a \neq b$.

Our main results are the following Theorems 1.1-1.4. All numerical computations are carried out using MATHEMATICA software.

Theorem 1.1 The double inequality

$$
\alpha_{1} A(a, b)+\left(1-\alpha_{1}\right) G(a, b)<S_{H A}(a, b)<\beta_{1} A(a, b)+\left(1-\beta_{1}\right) G(a, b)
$$

holds for all $a, b>0$ with $a \neq b$ if and only if $\alpha_{1} \leq 1 / 3$ and $\beta_{1} \geq 2 / \pi$.

Theorem 1.2 The two-sided inequality

$$
\alpha_{2} A(a, b)+\left(1-\alpha_{2}\right) Q(a, b)<S_{C A}(a, b)<\beta_{2} A(a, b)+\left(1-\beta_{2}\right) Q(a, b)
$$

holds true for all $a, b>0$ with $a \neq b$ if and only if $\alpha_{2} \geq 1 / 3$ and $\beta_{2} \leq[\sqrt{2} \log (2+\sqrt{3})-$ $\sqrt{3}] /[(\sqrt{2}-1) \log (2+\sqrt{3})]=0.2390 \ldots$.

Theorem 1.3 Let $\alpha_{3}, \beta_{3} \in[0,1 / 2]$, then the double inequality

$$
G\left[\alpha_{3} a+\left(1-\alpha_{3}\right) b, \alpha_{3} b+\left(1-\alpha_{3}\right) a\right]<S_{H A}(a, b)<G\left[\beta_{3} a+\left(1-\beta_{3}\right) b, \beta_{3} b+\left(1-\beta_{3}\right) a\right]
$$


holds for all $a, b>0$ with $a \neq b$ if and only if $\alpha_{3} \leq 1 / 2-\sqrt{6} / 6=0.09175 \ldots$ and $\beta_{3} \geq 1 / 2-$ $\sqrt{\pi^{2}-4} /(2 \pi)=0.1144 \ldots$.

Theorem 1.4 Let $\alpha_{4}, \beta_{4} \in[1 / 2,1]$, then the two-sided inequality

$$
Q\left[\alpha_{4} a+\left(1-\alpha_{4}\right) b, \alpha_{4} b+\left(1-\alpha_{4}\right) a\right]<S_{C A}(a, b)<Q\left[\beta_{4} a+\left(1-\beta_{4}\right) b, \beta_{4} b+\left(1-\beta_{4}\right) a\right]
$$

holds true for all $a, b>0$ with $a \neq b$ if and only if $\alpha_{4} \leq 1 / 2+\sqrt{6} / 6=0.9082 \ldots$ and $\beta_{4} \geq$ $1 / 2+\sqrt{3 /[\log (2+\sqrt{3})]^{2}-1} / 2=0.9271 \ldots$

\section{Two lemmas}

In order to prove our main results, we need two lemmas, which we present in this section.

Lemma 2.1 Let $p \in \mathbb{R}$ and

$$
f(x)=(1-p) x^{3}+\left(-2 p^{2}+5 p-1\right) x^{2}+\left(2 p^{2}+p-1\right) x+p-1 .
$$

Then the following statements are true:

(1) If $p=1 / 3$, then $f(x)<0$ for all $x \in(0,1)$ and $f(x)>0$ for all $x \in(1, \sqrt{2})$;

(2) If $p=2 / \pi$, then there exists $\lambda_{1} \in(0,1)$ such that $f(x)<0$ for $x \in\left(0, \lambda_{1}\right)$ and $f(x)>0$ for $x \in\left(\lambda_{1}, 1\right)$;

(3) If $p=[\sqrt{2} \log (2+\sqrt{3})-\sqrt{3}] /[(\sqrt{2}-1) \log (2+\sqrt{3})]$, then there exists $\lambda_{2} \in(1, \sqrt{2})$ such that $f(x)<0$ for $x \in\left(1, \lambda_{2}\right)$ and $f(x)>0$ for $x \in\left(\lambda_{2}, \sqrt{2}\right)$.

Proof For part (1), if $p=1 / 3$, then (2.1) becomes

$$
f(x)=\frac{2}{9}(x-1)\left(3 x^{2}+5 x+3\right) .
$$

Therefore, part (1) follows easily from (2.2).

For part (2), if $p=2 / \pi$, then simple computations lead to

$$
\begin{aligned}
& -2 p^{2}+5 p-1=\frac{-\pi^{2}+10 \pi-8}{\pi^{2}}>0, \\
& 2 p^{2}+p-1=\frac{-\pi^{2}+2 \pi+8}{\pi^{2}}>0, \\
& f(0)=-\frac{\pi-2}{\pi}<0, \\
& f(1)=\frac{2(6-\pi)}{\pi}>0, \\
& f^{\prime}(x)=3(1-p) x^{2}+2\left(-2 p^{2}+5 p-1\right) x+\left(2 p^{2}+p-1\right) .
\end{aligned}
$$

It follows from (2.3) and (2.4) together with (2.7) that $f(x)$ is strictly increasing on $(0,1)$. Therefore, part (2) follows from (2.5) and (2.6) together with the monotonicity of $f(x)$.

For part (3), if $p=[\sqrt{2} \log (2+\sqrt{3})-\sqrt{3}] /[(\sqrt{2}-1) \log (2+\sqrt{3})]=0.2390 \ldots$, then numerical computations lead to

$$
-2 p^{2}+5 p-1=0.0810 \ldots>0,
$$




$$
\begin{aligned}
& f(1)=-0.5656 \ldots<0, \\
& f(\sqrt{2})=0.6388 \ldots>0 .
\end{aligned}
$$

It follows from (2.7) and (2.8) that

$$
f^{\prime}(x)>3(1-p)+2\left(-2 p^{2}+5 p-1\right)+\left(2 p^{2}+p-1\right)=2 p(4-p)>0
$$

for $x \in(1, \sqrt{2})$.

Therefore, part (3) follows easily from (2.9)-(2.11).

Lemma 2.2 Let $p \in \mathbb{R}$ and

$$
\begin{aligned}
g(x)= & (2 p-1)^{4} x^{3}+\left(-256 p^{6}+768 p^{5}-1,008 p^{4}+736 p^{3}-296 p^{2}+56 p-3\right) x^{2} \\
& +\left(512 p^{6}-1,536 p^{5}+1,776 p^{4}-992 p^{3}+248 p^{2}-8 p-1\right) x \\
& +\left(-256 p^{6}+768 p^{5}-784 p^{4}+288 p^{3}-24 p^{2}+8 p-1\right) .
\end{aligned}
$$

Then the following statements are true:

(1) If $p=1 / 2-\sqrt{6} / 6$, then $g(x)<0$ for all $x \in(0,1)$;

(2) If $p=1 / 2+\sqrt{6} / 6$, then $g(x)>0$ for all $x \in(1,2)$;

(3) If $p=1 / 2-\sqrt{\pi^{2}-4} /(2 \pi)$, then there exists $\lambda_{3} \in(0,1)$ such that $g(x)<0$ for $x \in\left(0, \lambda_{3}\right)$ and $g(x)>0$ for $x \in\left(\lambda_{3}, 1\right)$;

(4) If $p=1 / 2+\sqrt{3 /[\log (2+\sqrt{3})]^{2}-1} / 2$, then there exists $\lambda_{4} \in(1,2)$ such that $g(x)<0$ for $x \in\left(1, \lambda_{4}\right)$ and $g(x)>0$ for $x \in\left(\lambda_{4}, 2\right)$.

Proof For parts (1) and (2), if $p=1 / 2-\sqrt{6} / 6$ or $p=1 / 2+\sqrt{6} / 6$, then (2.12) becomes

$$
g(x)=\frac{4}{27}(x-1)\left(3 x^{2}+4 x+2\right)
$$

Therefore, parts (1) and (2) follow from (2.13).

For part (3), if $p=1 / 2-\sqrt{\pi^{2}-4} /(2 \pi)$, then numerical computations show that

$$
\begin{aligned}
& -256 p^{6}+768 p^{5}-1,008 p^{4}+736 p^{3}-296 p^{2}+56 p-3 \\
& \quad=\frac{-3 \pi^{6}+56 \pi^{4}-240 \pi^{2}+256}{\pi^{6}}>0 \\
& 512 p^{6}-1,536 p^{5}+1,776 p^{4}-992 p^{3}+248 p^{2}-8 p-1 \\
& \quad=\frac{-\pi^{6}-8 \pi^{4}+240 \pi^{2}-512}{\pi^{6}}>0 \\
& g(0)=\frac{-\pi^{6}+8 \pi^{4}-16 \pi^{2}+256}{\pi^{6}}<0 \\
& g(1)=\frac{4\left(12-\pi^{2}\right)}{\pi^{2}}>0, \\
& g^{\prime}(x)=3(2 p-1)^{4} x^{2}+2\left(-256 p^{6}+768 p^{5}-1,008 p^{4}+736 p^{3}-296 p^{2}+56 p-3\right) x \\
& \quad+\left(512 p^{6}-1,536 p^{5}+1,776 p^{4}-992 p^{3}+248 p^{2}-8 p-1\right)
\end{aligned}
$$


From (2.14), (2.15) and (2.18) we clearly see that $g(x)$ is strictly increasing on $(0,1)$. Therefore, part (3) follows from (2.16) and (2.17) together with the monotonicity of $g(x)$.

For part (4), if $p=1 / 2+\sqrt{3 /[\log (2+\sqrt{3})]^{2}-1} / 2$, then numerical computations lead to

$$
\begin{aligned}
& -256 p^{6}+768 p^{5}-1,008 p^{4}+736 p^{3}-296 p^{2}+56 p-3=-0.2329 \ldots<0, \\
& 512 p^{6}-1,536 p^{5}+1,776 p^{4}-992 p^{3}+248 p^{2}-8 p-1=-0.6027 \ldots<0, \\
& g(1)=-0.7567 \ldots<0, \\
& g(2)=1.6692 \ldots>0, \\
& -48 p^{4}+96 p^{3}-68 p^{2}+20 p-1=0.1322 \ldots>0 .
\end{aligned}
$$

It follows from (2.18), (2.19), (2.20) and (2.23) that

$$
\begin{aligned}
g^{\prime}(x)> & 3(2 p-1)^{4} x^{2}+2\left(-256 p^{6}+768 p^{5}-1,008 p^{4}+736 p^{3}-296 p^{2}+56 p-3\right) x^{2} \\
& +\left(512 p^{6}-1,536 p^{5}+1,776 p^{4}-992 p^{3}+248 p^{2}-8 p-1\right) x^{2} \\
= & 4\left(-48 p^{4}+96 p^{3}-68 p^{2}+20 p-1\right) x^{2}>0
\end{aligned}
$$

for $x \in(1,2)$.

Therefore, part (4) follows from (2.21) and (2.22) together with (2.24).

\section{Proofs of Theorems 1.1-1.4}

Proof of Theorem 1.1 Without loss of generality, we assume that $a>b$. Let $v=(a-b) /(a+b)$, $\lambda=v \sqrt{2-v^{2}}, x=\sqrt[4]{1-\lambda^{2}}$ and $p \in\{1 / 3,2 / \pi\}$. Then $v, \lambda, x \in(0,1)$ and (1.4) leads to

$$
\begin{aligned}
& \frac{S_{H A}(a, b)-G(a, b)}{A(a, b)-G(a, b)}=\frac{\lambda-\left(1-\lambda^{2}\right)^{1 / 4} \sin ^{-1}(\lambda)}{\left[1-\left(1-\lambda^{2}\right)^{1 / 4}\right] \sin ^{-1}(\lambda)}, \\
& S_{H A}(a, b)-[p A(a, b)+(1-p) G(a, b)] \\
& =A(a, b)\left[\frac{\lambda}{\sin ^{-1}(\lambda)}-(1-p)\left(1-\lambda^{2}\right)^{1 / 4}-p\right] \\
& =\frac{A(a, b)\left[p+(1-p)\left(1-\lambda^{2}\right)^{1 / 4}\right]}{\sin ^{-1}(\lambda)} F(x),
\end{aligned}
$$

where

$$
\begin{aligned}
& F(x)=\frac{\sqrt{1-x^{4}}}{(1-p) x+p}-\sin ^{-1}\left(\sqrt{1-x^{4}}\right), \\
& F(0)=\frac{1}{p}-\frac{\pi}{2} \\
& F(1)=0
\end{aligned}
$$

and

$$
F^{\prime}(x)=\frac{1-x}{\sqrt{1-x^{4}}[(1-p) x+p]^{2}} f(x),
$$

where $f(x)$ is defined as in Lemma 2.1. 
We divide the proof into two cases.

Case 1: $p=1 / 3$. Then from Lemma 2.1(1) and (3.5) we clearly see that $F(x)$ is strictly decreasing on $(0,1)$. Therefore,

$$
S_{H A}(a, b)>\frac{1}{3} A(a, b)+\frac{2}{3} G(a, b)
$$

for all $a, b>0$ with $a \neq b$ follows from (3.2) and (3.4) together with the monotonicity of $F(x)$.

Case 2: $p=2 / \pi$. Then from (3.3), (3.5) and Lemma 2.1(2) we know that

$$
F(0)=0
$$

and there exists $\lambda_{1} \in(0,1)$ such that $F(x)$ is strictly decreasing on $\left(0, \lambda_{1}\right]$ and strictly increasing on $\left[\lambda_{1}, 1\right)$. Therefore,

$$
S_{H A}(a, b)<\frac{2}{\pi} A(a, b)+\left(1-\frac{2}{\pi}\right) G(a, b)
$$

for all $a, b>0$ with $a \neq b$ follows from (3.2) and (3.4) together with (3.7) and the piecewise monotonicity of $F(x)$.

Note that

$$
\lim _{\lambda \rightarrow 0^{+}} \frac{\lambda-\left(1-\lambda^{2}\right)^{1 / 4} \sin ^{-1}(\lambda)}{\left[1-\left(1-\lambda^{2}\right)^{1 / 4}\right] \sin ^{-1}(\lambda)}=\frac{1}{3}
$$

and

$$
\lim _{\lambda \rightarrow 1^{-}} \frac{\lambda-\left(1-\lambda^{2}\right)^{1 / 4} \sin ^{-1}(\lambda)}{\left[1-\left(1-\lambda^{2}\right)^{1 / 4}\right] \sin ^{-1}(\lambda)}=\frac{2}{\pi} .
$$

Therefore, Theorem 1.1 follows from (3.6) and (3.8) together with the following statements.

- If $\alpha>1 / 3$, then equations (3.1) and (3.9) imply that there exists small enough $\delta>0$ such that $S_{H A}(a, b)<\alpha A(a, b)+(1-\alpha) G(a, b)$ for all $a>b>0$ with $b / a \in(1-\delta, 1)$.

- If $\beta<2 / \pi$, then equations (3.1) and (3.10) imply that there exists large enough $M>1$ such that $S_{H A}(a, b)>\beta A(a, b)+(1-\beta) G(a, b)$ for all $a>b>0$ with $a / b \in(M,+\infty)$.

Proof of Theorem 1.2 Without loss of generality, we assume that $a>b$. Let $v=(a-b) /(a+$ b), $\mu=v \sqrt{2+v^{2}}, x=\sqrt[4]{1+\mu^{2}}$ and $p \in\{[\sqrt{2} \log (2+\sqrt{3})-\sqrt{3}] /[(\sqrt{2}-1) \log (2+\sqrt{3})], 1 / 3\}$. Then $v \in(0,1), \mu \in(0, \sqrt{3}), x \in(1, \sqrt{2})$ and (1.5) leads to

$$
\begin{aligned}
& \frac{S_{C A}(a, b)-Q(a, b)}{A(a, b)-Q(a, b)}=\frac{\mu-\left(1+\mu^{2}\right)^{1 / 4} \sinh ^{-1}(\mu)}{\left[1-\left(1+\mu^{2}\right)^{1 / 4}\right] \sinh ^{-1}(\mu)}, \\
& S_{C A}(a, b)-[p A(a, b)+(1-p) Q(a, b)] \\
& =A(a, b)\left[\frac{\mu}{\sinh ^{-1}(\mu)}-(1-p)\left(1+\mu^{2}\right)^{1 / 4}-p\right] \\
& =\frac{A(a, b)\left[(1-p)\left(1+\mu^{2}\right)^{1 / 4}+p\right]}{\sinh ^{-1}(\mu)} G(x),
\end{aligned}
$$


where

$$
\begin{aligned}
& G(x)=\frac{\sqrt{x^{4}-1}}{(1-p) x+p}-\sinh ^{-1}\left(\sqrt{x^{4}-1}\right), \\
& G(1)=0, \\
& G(\sqrt{2})=\frac{\sqrt{3}}{\sqrt{2}-(\sqrt{2}-1) p}-\log (2+\sqrt{3}), \\
& G^{\prime}(x)=\frac{x-1}{\sqrt{x^{4}-1}[(1-p) x+p]^{2}} f(x),
\end{aligned}
$$

where $f(x)$ is defined as in Lemma 2.1.

We divide the proof into two cases.

Case 1: $p=[\sqrt{2} \log (2+\sqrt{3})-\sqrt{3}] /[(\sqrt{2}-1) \log (2+\sqrt{3})]=0.2390 \ldots$ Then from (3.14) and (3.15) together with Lemma 2.1(3) we clearly see that there exists $\lambda_{2} \in(1, \sqrt{2})$ such that $G(x)$ is strictly decreasing on $\left(1, \lambda_{2}\right]$ and strictly increasing on $\left[\lambda_{2}, \sqrt{2}\right)$, and

$$
G(\sqrt{2})=0
$$

Therefore,

$$
\begin{aligned}
S_{C A}(a, b)< & \frac{\sqrt{2} \log (2+\sqrt{3})-\sqrt{3}}{(\sqrt{2}-1) \log (2+\sqrt{3})} A(a, b) \\
& +\frac{\sqrt{3}-\log (2+\sqrt{3})}{(\sqrt{2}-1) \log (2+\sqrt{3})} Q(a, b)
\end{aligned}
$$

for all $a, b>0$ with $a \neq b$ follows easily from (3.12) and (3.13) together with (3.16) and the piecewise monotonicity of $G(x)$.

Case 2: $p=1 / 3$. Then Lemma 2.1(1) and (3.15) lead to the conclusion that $G(x)$ is strictly increasing on $(1, \sqrt{2})$. Therefore,

$$
S_{C A}(a, b)>\frac{1}{3} A(a, b)+\frac{2}{3} Q(a, b)
$$

for all $a, b>0$ with $a \neq b$ follows from (3.12) and (3.13) together with the monotonicity of $G(x)$.

Note that

$$
\lim _{\mu \rightarrow 0^{+}} \frac{\mu-\left(1+\mu^{2}\right)^{1 / 4} \sinh ^{-1}(\mu)}{\left[1-\left(1+\mu^{2}\right)^{1 / 4}\right] \sinh ^{-1}(\mu)}=\frac{1}{3}
$$

and

$$
\lim _{\mu \rightarrow \sqrt{3}^{-}} \frac{\mu-\left(1+\mu^{2}\right)^{1 / 4} \sinh ^{-1}(\mu)}{\left[1-\left(1+\mu^{2}\right)^{1 / 4}\right] \sinh ^{-1}(\mu)}=\frac{\sqrt{2} \log (2+\sqrt{3})-\sqrt{3}}{(\sqrt{2}-1) \log (2+\sqrt{3})} .
$$

Therefore, Theorem 1.2 follows from (3.11) and (3.17)-(3.20). 
Proof of Theorem 1.3 Without loss of generality, we assume that $a>b$. Let $v=(a-b) /(a+$ $b), \lambda=v \sqrt{2-v^{2}}, x=\sqrt{1-\lambda^{2}}$ and $p \in[0,1 / 2]$. Then $v, \lambda, x \in(0,1)$ and (1.4) leads to

$$
\begin{aligned}
G & {[p a+(1-p) b, p b+(1-p) a]-S_{H A}(a, b) } \\
& =A(a, b)\left[\sqrt{1-(1-2 p)^{2}\left(1-\sqrt{1-\lambda^{2}}\right)}-\frac{\lambda}{\sin ^{-1}(\lambda)}\right] \\
& =\frac{A(a, b) \sqrt{1-(1-2 p)^{2}\left(1-\sqrt{1-\lambda^{2}}\right)}}{\sin ^{-1}(\lambda)} H(x),
\end{aligned}
$$

where

$$
\begin{aligned}
& H(x)=\sin ^{-1}\left(\sqrt{1-x^{2}}\right)-\frac{\sqrt{1-x^{2}}}{\sqrt{(1-2 p)^{2} x-(1-2 p)^{2}+1}}, \\
& H(1)=0 \\
& H(0)=\frac{\pi}{2}-\frac{1}{\sqrt{1-(1-2 p)^{2}}}
\end{aligned}
$$

and

$$
H^{\prime}(x)=\frac{h(x)}{2 \sqrt{1-x^{2}}\left[(1-2 p)^{2} x-(1-2 p)^{2}+1\right]^{3 / 2}},
$$

where

$$
\begin{aligned}
& h(x) \\
& \quad=(1-2 p)^{2} x^{2}+2\left[1-(1-2 p)^{2}\right] x+(1-2 p)^{2}-2\left[(1-2 p)^{2} x-(1-2 p)^{2}+1\right]^{3 / 2} \\
& \quad=\frac{(x-1) g(x)}{(1-2 p)^{2} x^{2}+2\left[1-(1-2 p)^{2}\right] x+(1-2 p)^{2}+2\left[(1-2 p)^{2} x-(1-2 p)^{2}+1\right]^{3 / 2}},
\end{aligned}
$$

where $g(x)$ is defined as in Lemma 2.2.

We divide the proof into four cases.

Case 1: $p=1 / 2-\sqrt{6} / 6$. Then Lemma 2.2(1) and (3.24) together with (3.25) lead to the conclusion that $H(x)$ is strictly increasing on $(0,1)$. Therefore,

$$
S_{H A}(a, b)>G\left[\left(\frac{1}{2}-\frac{\sqrt{6}}{6}\right) a+\left(\frac{1}{2}+\frac{\sqrt{6}}{6}\right) b,\left(\frac{1}{2}-\frac{\sqrt{6}}{6}\right) b+\left(\frac{1}{2}+\frac{\sqrt{6}}{6}\right) a\right]
$$

for all $a, b>0$ with $a \neq b$ follows easily from (3.21) and (3.22) together with the monotonicity of $H(x)$.

Case 2: $1 / 2-\sqrt{6} / 6<p \leq 1 / 2$. Let $q=(1-2 p)^{2}$ and $\lambda \rightarrow 0^{+}$, then $0 \leq q<2 / 3$ and power series expansions lead to

$$
\begin{aligned}
& \sqrt{1-(1-2 p)^{2}\left(1-\sqrt{1-\lambda^{2}}\right)}-\frac{\lambda}{\sin ^{-1} \lambda} \\
& =\frac{\sqrt{1-q\left(1-\sqrt{1-\lambda^{2}}\right)} \sin ^{-1} \lambda-\lambda}{\sin ^{-1} \lambda}=\frac{1}{\sin ^{-1} \lambda}\left[\left(\frac{1}{6}-\frac{q}{4}\right) \lambda^{3}+o\left(\lambda^{3}\right)\right] .
\end{aligned}
$$

Equations (3.21) and (3.26) imply that there exists small enough $\delta_{1}>0$ such that $S_{H A}(a, b)<G[p a+(1-p) b, p b+(1-p) a]$ for all $a, b>0$ with $b / a \in\left(1-\delta_{1}, 1\right)$. 
Case 3: $p=1 / 2-\sqrt{\pi^{2}-4} /(2 \pi)$. Then from Lemma 2.2(3) and (3.23)-(3.25) we clearly see that there exists $\lambda_{3} \in(0,1)$ such that $H(x)$ is strictly increasing on $\left(0, \lambda_{3}\right]$ and strictly decreasing on $\left[\lambda_{3}, 1\right)$, and

$$
H(0)=0 \text {. }
$$

Therefore,

$$
\begin{aligned}
S_{H A}(a, b)< & G\left[\left(\frac{1}{2}-\frac{\sqrt{\pi^{2}-4}}{2 \pi}\right) a+\left(\frac{1}{2}+\frac{\sqrt{\pi^{2}-4}}{2 \pi}\right) b,\right. \\
& \left.\left(\frac{1}{2}-\frac{\sqrt{\pi^{2}-4}}{2 \pi}\right) b+\left(\frac{1}{2}+\frac{\sqrt{\pi^{2}-4}}{2 \pi}\right) a\right]
\end{aligned}
$$

for all $a, b>0$ with $a \neq b$ follows easily from (3.21) and (3.22) together with (3.27) and the piecewise monotonicity of $H(x)$.

Case 4: $0 \leq p<1 / 2-\sqrt{\pi^{2}-4} /(2 \pi)$. Then

$$
\lim _{\lambda \rightarrow 1^{-}}\left[\sqrt{1-(1-2 p)^{2}\left(1-\sqrt{1-\lambda^{2}}\right)}-\frac{\lambda}{\sin ^{-1}(\lambda)}\right]=\sqrt{1-(1-2 p)^{2}}-\frac{2}{\pi}<0 .
$$

Equation (3.21) and inequality (3.28) imply that there exists large enough $M_{1}>1$ such that $S_{H A}(a, b)>G[p a+(1-p) b, p b+(1-p) a]$ for all $a, b>0$ with $a / b \in\left(M_{1},+\infty\right)$.

Proof of Theorem 1.4 Without loss of generality, we assume that $a>b$. Let $v=(a-b) /(a+$ $b), \mu=v \sqrt{2+v^{2}}, x=\sqrt{1+\mu^{2}}$ and $p \in[1 / 2,1]$. Then $v \in(0,1), \mu \in(0, \sqrt{3}), x \in(1,2)$ and (1.5) leads to

$$
\begin{aligned}
& Q[p a+(1-p) b, p b+(1-p) a]-S_{C A}(a, b) \\
& =A(a, b)\left[\sqrt{1+(1-2 p)^{2}\left(\sqrt{1+\mu^{2}}-1\right)}-\frac{\mu}{\sinh ^{-1}(\mu)}\right] \\
& =\frac{A(a, b) \sqrt{1+(1-2 p)^{2}\left(\sqrt{1+\mu^{2}}-1\right)}}{\sinh ^{-1}(\mu)} J(x),
\end{aligned}
$$

where

$$
\begin{aligned}
J(x)= & \sinh ^{-1}\left(\sqrt{x^{2}-1}\right)-\frac{\sqrt{x^{2}-1}}{\sqrt{(1-2 p)^{2} x-(1-2 p)^{2}+1}}, \\
J(1)= & , \\
J(2)= & \log (2+\sqrt{3})-\frac{\sqrt{3}}{\sqrt{1+(1-2 p)^{2}}}, \\
J^{\prime}(x)= & \frac{2\left[(1-2 p)^{2} x-(1-2 p)^{2}+1\right]^{3 / 2}-\left[(1-2 p)^{2} x^{2}+2\left(1-(1-2 p)^{2}\right) x+(1-2 p)^{2}\right]}{2 \sqrt{x^{2}-1}\left[(1-2 p)^{2} x-(1-2 p)^{2}+1\right]^{3 / 2}} \\
= & -\frac{1}{2\left[(1-2 p)^{2} x-(1-2 p)^{2}+1\right]^{3 / 2}+\left[(1-2 p)^{2} x^{2}+2\left(1-(1-2 p)^{2}\right) x+(1-2 p)^{2}\right]} \\
& \times \frac{x-1}{2 \sqrt{x^{2}-1}\left[(1-2 p)^{2} x-(1-2 p)^{2}+1\right]^{3 / 2}} g(x),
\end{aligned}
$$

where $g(x)$ is defined as in Lemma 2.2. 
We divide the proof into four cases.

Case 1: $p=1 / 2+\sqrt{6} / 6$. Then Lemma 2.2(2) and (3.32) lead to the conclusion that $J(x)$ is strictly increasing on $(1,2)$. Therefore,

$$
S_{C A}(a, b)>Q\left[\left(\frac{1}{2}+\frac{\sqrt{6}}{6}\right) a+\left(\frac{1}{2}-\frac{\sqrt{6}}{6}\right) b,\left(\frac{1}{2}+\frac{\sqrt{6}}{6}\right) b+\left(\frac{1}{2}-\frac{\sqrt{6}}{6}\right) a\right]
$$

for all $a, b>0$ with $a \neq b$ follows easily from (3.29) and (3.30) together with the monotonicity of $J(x)$.

Case $2: 1 / 2+\sqrt{6} / 6<p \leq 1$. Let $q=(1-2 p)^{2}$ and $\mu \rightarrow 0^{+}$, then $1 \geq q>2 / 3$ and power series expansions lead to

$$
\begin{gathered}
\sqrt{1+(1-2 p)^{2}\left(\sqrt{1+\mu^{2}}-1\right)}-\frac{\mu}{\sinh ^{-1}(\mu)} \\
=\frac{\sqrt{1+q\left(\sqrt{1+\mu^{2}}-1\right)} \sinh ^{-1}(\mu)-\mu}{\sinh ^{-1}(\mu)} \\
=\frac{1}{\sinh ^{-1}(\mu)}\left[\left(\frac{1}{4} q-\frac{1}{6}\right) \mu^{3}+o\left(\mu^{3}\right)\right] .
\end{gathered}
$$

Equations (3.29) and (3.33) imply that there exists small enough $\delta_{2}>0$ such that $S_{C A}(a, b)<Q[p a+(1-p) b, p b+(1-p) a]$ for all $a, b>0$ with $b / a \in\left(1-\delta_{2}, 1\right)$.

Case 3: $p=1 / 2+\sqrt{3 /[\log (2+\sqrt{3})]^{2}-1} / 2$. Then (3.31) and (3.32) together with Lemma 2.2(4) lead to the conclusion that there exists $\lambda_{4} \in(1,2)$ such that $J(x)$ is strictly increasing on $\left(1, \lambda_{4}\right]$ and strictly decreasing on $\left[\lambda_{4}, 2\right)$, and

$$
J(2)=0 .
$$

Therefore,

$$
S_{C A}(a, b)<Q[p a+(1-p) b, p b+(1-p) a]
$$

for all $a, b>0$ with $a \neq b$ follows easily from (3.29) and (3.30) together with (3.34) and the piecewise monotonicity of $J(x)$.

Case $4: 1 / 2 \leq p<1 / 2+\sqrt{3 /[\log (2+\sqrt{3})]^{2}-1} / 2$. Then

$$
\begin{aligned}
& \lim _{\mu \rightarrow \sqrt{3}^{-}}\left[\sqrt{1+(1-2 p)^{2}\left(\sqrt{1+\mu^{2}}-1\right)}-\frac{\mu}{\sinh ^{-1}(\mu)}\right] \\
& =\sqrt{1+(2 p-1)^{2}}-\frac{\sqrt{3}}{\log (2+\sqrt{3})}<0 .
\end{aligned}
$$

Equation (3.29) and inequality (3.35) imply that there exists large enough $M_{2}>1$ such that $S_{C A}(a, b)>Q[p a+(1-p) b, p b+(1-p) a]$ for all $a, b>0$ with $a / b \in\left(M_{2},+\infty\right)$. 
Authors' contributions

W-MQ provided the main idea and carried out the proof of Lemmas 2.1 and 2.2. Y-MC carried out the proof of Theorems 1.1-1.4. All authors read and approved the final manuscript.

\section{Author details}

${ }^{1}$ School of Distance Education, Huzhou Broadcast and TV University, Huzhou, 313000, China. ${ }^{2}$ School of Mathematics and Computation Science, Hunan City University, Yiyang, 413000, China.

\section{Acknowledgements}

The authors would like to express their deep gratitude to the referees for giving many valuable suggestions. The research was supported by the Natural Science Foundation of China under Grants 61374086 and 11171307, the Natural Science Foundation of the Open University of China under Grant Q1601E-Y and the Natural Science Foundation of Zhejiang Broadcast and TV University under Grant XKT-13Z04.

Received: 16 January 2014 Accepted: 16 April 2014 Published: 12 May 2014

\section{References}

1. Carlson, BC: Algorithms involving arithmetic and geometric means. Am. Math. Mon. 78, 496-505 (1971)

2. Borwein, JM, Borwein, PB: Pi and the AGM: A Study in Analytic Number Theory and Computational Complexity. Wiley, New York (1987)

3. Neuman, E, Sándor, J: On the Schwab-Borchardt mean. Math. Pannon. 14(2), 253-266 (2003)

4. Neuman, E, Sándor, J: On the Schwab-Borchardt mean II. Math. Pannon. 17(1), 49-59 (2006)

5. Neuman, E: A note on a certain bivariate mean. J. Math. Inequal. 6(4), 637-643 (2012)

6. Chu, Y-M, Long, B-Y: Bounds of the Neuman-Sándor mean using power and identric mean. Abstr. Appl. Anal. 2013, Article ID 832591 (2013)

7. Zhao, T-H, Chu, Y-M, Liu, B-Y: Optimal bounds for Neuman-Sándor mean in terms of the convex combinations of harmonic, geometric, quadratic, and contraharmonic means. Abstr. Appl. Anal. 2013, Article ID 302635 (2013)

8. Neuman, E: On some means derived from the Schwab-Borchardt mean. J. Math. Inequal. 8(1), 171-183 (2014)

9. Neuman, E: On some means derived from the Schwab-Borchardt mean II. J. Math. Inequal. 8(2), 361-370 (2014)

10. He, Z-Y, Chu, Y-M, Wang, M-K: Optimal bounds for Neuman means in terms of harmonic and contraharmonic means. J. Appl. Math. 2013, Article ID 807623 (2013)

10.1186/1029-242X-2014-175

Cite this article as: Qian and Chu: Optimal bounds for Neuman means in terms of geometric, arithmetic and quadratic means. Journal of Inequalities and Applications 2014, 2014:175

\section{Submit your manuscript to a SpringerOpen ${ }^{\circ}$ journal and benefit from:}

- Convenient online submission

- Rigorous peer review

- Immediate publication on acceptance

- Open access: articles freely available online

- High visibility within the field

- Retaining the copyright to your article 